\title{
Improved Efficacy of Transcatheter Arterial Chemoembolization Using Warmed Miriplatin for Hepatocellular Carcinoma
}

\author{
Daisuke Yasui, ${ }^{1}$ Satoru Murata, ${ }^{1}$ Shiro Onozawa, ${ }^{1}$ Takahiko Mine, ${ }^{1}$ Tatsuo Ueda, ${ }^{1}$ \\ Fumie Sugihara, ${ }^{1}$ Chiaki Kawamoto, ${ }^{2}$ Eiji Uchida, ${ }^{3}$ and Shin-ichiro Kumita ${ }^{1}$ \\ ${ }^{1}$ Department of Radiology/Center for Advanced Medical Technology, Nippon Medical School, 1-1-5 Sendagi, Bunkyo-ku, \\ Tokyo 113-8603, Japan \\ ${ }^{2}$ Division of Gastroenterology and Hepatology, Department of Internal Medicine, Nippon Medical School, 1-1-5 Sendagi, \\ Bunkyo-ku, Tokyo 113-8603, Japan \\ ${ }^{3}$ Department of Surgery, Nippon Medical School, 1-1-5 Sendagi, Bunkyo-ku, Tokyo 113-8603, Japan
}

Correspondence should be addressed to Daisuke Yasui; ledhotcp@nms.ac.jp

Received 25 June 2014; Accepted 17 August 2014; Published 8 September 2014

Academic Editor: Pascal Niggemann

Copyright (C) 2014 Daisuke Yasui et al. This is an open access article distributed under the Creative Commons Attribution License, which permits unrestricted use, distribution, and reproduction in any medium, provided the original work is properly cited.

\begin{abstract}
The aim of this study was to evaluate the efficacy and safety of transcatheter arterial chemoembolization (TACE) using warmed and nonwarmed miriplatin for hepatocellular carcinoma. Eighty patients (117 nodules), treated between January 2010 and June 2013, were evaluated. Thirty-two and 85 nodules were treated with nonwarmed and warmed miriplatin, respectively. The efficacy of TACE was evaluated on a per nodule basis according to treatment effect (TE). Adverse events were evaluated according to the Common Terminology Criteria for Adverse Events (CTCAE) v4.0. TE grades were significantly improved in the warmed group compared to the nonwarmed group (nonwarmed: TE 4, 12.5\%; TE 3, 0\%; TE 2, 15.6\%; TE 1, 71.9\%; warmed: TE 4, 34.1\%; TE 3, 5.9\%; TE 2, 9.4\%; TE $1,50.6 \% ; P=0.017)$. Multivariate analysis revealed significant impact of warming miriplatin on objective response rate (odds ratio, 12.35; 95\% confidence interval, 2.90-90.0; $P=0.0028$ ). CTCAE grades of elevated aspartate and alanine transaminase after TACE were significantly higher in the warmed group $(P=0.0083$ and 0.0068 , resp.); however, all adverse events were only transient. The use of warmed miriplatin in TACE significantly improved TE without causing serious complications.
\end{abstract}

\section{Introduction}

Transcatheter arterial chemoembolization (TACE) is a standard therapy for intermediate stage unresectable hepatocellular carcinoma (HCC) [1]. Previous randomized controlled studies have shown that TACE prolongs survival and controls symptoms of HCC $[2,3]$.

Doxorubicin, epirubicin, cisplatin, and mitomycin C have been widely used as chemotherapeutic agents, either alone or in combination [4]. It is known that epirubicin, cisplatin, and mitomycin $\mathrm{C}$ can cause arteritis after injection, leading to hepatic artery occlusion and development of extrahepatic collateral pathways [5]. This change in vascular anatomy can make repetitive TACE difficult and limits the long-term efficacy of TACE.

A new platinum agent, miriplatin $((S P-4-2)-[(1 R, 2 R)-$ cyclohexane-1,2-diamine- $\left.N, N^{\prime}\right]$ bis (tetradecanoato-O) platinum monohydrate; Dainippon Sumitomo Pharma, Osaka, Japan), was recently developed [6,7]. It is a lipophilic platinum complex that can be easily suspended in Lipiodol (Guerbet, Aulnay-sous-Bois, France) and gradually releases active platinum compounds in tumor tissue [7]. According to the experience in our facility (296 sessions of TACE using miriplatin in the past 4 years), miriplatin is likely to cause minimal damage to the hepatic artery (unpublished data). Thus, TACE using miriplatin can be performed repeatedly as needed [8].

Another advantage of miriplatin is its less severe toxicity profile compared to other agents, resulting from gradual release of platinum into serum $[9,10]$.

Less damage to feeding arteries and less severe adverse effects make miriplatin suitable for TACE; however, when compared to other agents, the clinical outcomes of TACE using miriplatin have not been satisfactory [11-13]. It has 
been reported that the high viscosity and the large oil droplet of miriplatin can cause early occlusion of feeding vessels, leading to inadequate accumulation in tumors [11]. The viscosity of miriplatin suspension has been shown to decrease as temperature increases, dropping from $50 \mathrm{mPa} \cdot \mathrm{s}$ at $25^{\circ} \mathrm{C}$ to $12 \mathrm{mPa} \cdot \mathrm{s}$ at $60^{\circ} \mathrm{C}[14,15]$. Therefore, local tumor control could be improved by using warmed miriplatin in TACE, as warming miriplatin will reduce its viscosity and increase miriplatin accumulation in the tumor.

Thus, the purpose of this study was to evaluate and compare the efficacy of nonwarmed miriplatin versus warmed miriplatin in TACE and to review the adverse events in both treatment groups.

\section{Materials and Methods}

This study was approved by the local institutional review board. The review board waived the need for informed consent given the retrospective design of the study.

2.1. Patients. Patients were eligible for this retrospective study if they were diagnosed with HCC by either contrast-enhanced dynamic computed tomography or dynamic magnetic resonance imaging using gadolinium ethoxybenzyl diethylenetriamine pentaacetic acid. A total of 100 patients (140 nodules) were initially selected and met all the following requirements: 1 nodule per hepatic segment and a well-demarcated and hypervascular lesion (Figure 1). Among those, 20 patients (23 nodules) were excluded for the following reasons: 6 patients ( 7 nodules) for difficulty in selective catheterization of the feeding artery (beyond the second branch of the proper hepatic artery); 9 patients (11 nodules) for massive arterioportal shunts (A-P shunt) in which a segmental portal vein was visualized on hepatic arteriography; and 5 patients ( 5 nodules) for inadequate miriplatin accumulation in the target lesion because of large tumor size or anastomosis with vital vessels (pericardial and pulmonary veins) (Figure 1).

A total of 80 patients (117 nodules) were finally selected (Figure 1). TACE with nonwarmed miriplatin was performed on 22 patients (32 nodules) between January 2010 and December 2010. This included 17 men and 5 women, with a median age of 68 years (range, $51-83$ years) (Table 1 ). TACE using warmed miriplatin was initiated in January 2011 in order to improve local tumor control by reducing viscosity. Fifty-eight patients (85 nodules) were treated with warmed miriplatin between January 2011 and June 2013. This included 34 male and 24 female patients, with a median age of 73 years (range, 50-91 years) (Table 1).

2.2. Treatment Procedure. The entire treatment procedure was performed under local anesthesia by administering lidocaine subcutaneously. A 4-French sheath (Super Sheath; Medikit, Miyazaki, Japan) was inserted via the femoral artery. Feeding arteries were routinely selected beyond the second branch of the proper hepatic artery and were cannulated with 2.0-French microcatheters (Gold Crest-MRT; Koshin medical, Tokyo, Japan). The miriplatin suspension was prepared by directly mixing miriplatin powder with lipiodol. The miriplatin/lipiodol suspension was prepared at $25^{\circ} \mathrm{C}$ for the nonwarmed miriplatin group. For preparing warmed miriplatin, lipiodol was mixed with miriplatin powder first and then the miriplatin/lipiodol suspension was immersed in a hot water bath for more than 5 minutes, which was kept at $55^{\circ} \mathrm{C}$ as measured using a thermometer inside a clean container placed in an electric range. The stability of miriplatin/lipiodol suspension at this temperature was confirmed by the manufacturer. The standard full dosage of the TACE protocol was miriplatin $120 \mathrm{mg}$. The miriplatin/lipiodol suspension was administered slowly under fluoroscopic guidance immediately after preparation without causing reflux, until the vascular bed of the target nodule was fully filled with the suspension, as confirmed under fluoroscopy. Thus, the amount of the miriplatin/lipiodol suspension was not predetermined, but rather decided by angiographic findings. Finally, the feeding arteries were embolized with ready-made $2 \mathrm{~mm}$ pieces of gelatin sponge (Gelpart; Nippon Kayaku, Tokyo, Japan), until complete stasis of the feeding arteries was obtained.

2.3. Evaluation of Treatment Effect. The response to TACE was evaluated on a per nodule basis, according to the 4-grade system: treatment effect (TE) grades 1-4 [16]. Pretreatment nodule size was measured using the most recent image (within 3 months) prior to the first treatment. The product of long and short axes length in the maximum cross section was calculated for each nodule before and after TACE, and nodules were classified according to the change in size: grade 4 (TE 4 ), 100\% reduction in size or complete tumor necrosis; grade 3 (TE 3 ), $50 \%$ to $100 \%$ reduction in size; grade 2 (TE $2),<50 \%$ reduction to $<25 \%$ increase in size; and grade 1 (TE 1), more than $25 \%$ increase in size. Objective response rate (ORR), defined as the proportion of TE 3 and 4 cases to the total, and disease control rate (DCR), defined as the proportion of TE 2, 3, and 4 cases to the total, were compared between the nonwarmed and warmed miriplatin groups. The dose of miriplatin used in each session was also compared between the 2 groups. Multivariate analysis was performed to identify factors that had significant influence on ORR.

\subsection{Evaluation of the Effect of Anticancer Agents on the Hepatic} Artery. Arterial damage to the hepatic artery was defined as vessel irregularity, stenosis, or occlusion. It was evaluated on a per regimen basis, independent of other analyses. All patients were assigned to three groups according to anticancer agent used in TACE: epirubicin/cisplatin, nonwarmed miriplatin, and warmed miriplatin, considering both past treatment and the treatment included in this study. Therefore, patients with multiple treatment history using different agents were assigned to more than one group. Preoperative arterial damage was evaluated with celiac arteriography prior to the treatment using each agent. Postoperative celiac arteriography before the usage of other agents was compared with the preoperative one. Damage to the hepatic artery was evaluated according to the 5-grade system: grade 0 , no obvious damage; grade 1, irregular vessel wall; grade 2 , vessel narrowing; grade 3 , stenosis; and grade 4, occlusion. Severe damage was defined 


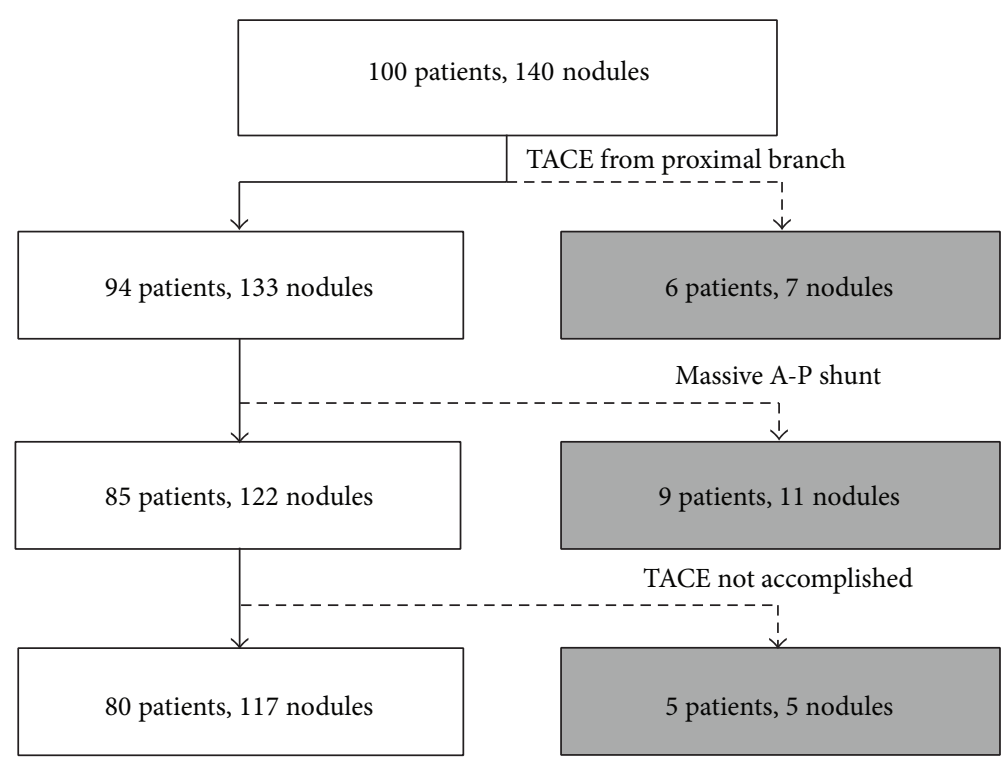

FIGURE 1: Patient enrollment. Gray boxes indicate patients excluded from the study. TACE: transcatheter arterial chemoembolization; A-P: arterioportal.

as grade 3 and grade 4 . The level of arterial damage was classified into 4 levels: level 1, the proper hepatic artery; level 2, lobar branches; level 3, segmental branches; and level 4, subsegmental branches. Development of A-P shunt was also evaluated. The evaluation was performed by two observers (D.Y and T.M), independently and blinded to each other. After individual evaluation, the findings were disclosed and discrepancy in the findings was discussed by 2 observers.

2.5. Follow-Up. Either contrast-enhanced computed tomography or magnetic resonance imaging was performed every 3 to 6 months after TACE. The end of the follow-up period was defined as either the last patient visit or the addition of other treatments: TACE with other agents, radiofrequency ablation, or surgery. The entire follow-up period was completed in September 2013.

2.6. Adverse Events. Common Terminology Criteria for Adverse Events (CTCAE) version 4.0 was used to evaluate the safety of TACE using warmed miriplatin. Adverse events were evaluated on a per treatment session basis. Levels of aspartate transaminase (AST), alanine transaminase (ALT), total bilirubin, and complete blood counts were measured, and pre- and postoperative values were compared between the 2 groups. Eosinophilia, which is among the characteristic adverse events of miriplatin (defined as more than 450 cells $/ \mu \mathrm{L}$ ), was also evaluated. Data obtained closest to the first treatment (within 1 month) were used as preoperative values. Either peak or trough data after the procedure were evaluated. Abnormal values were monitored to check for their return to the baseline. Incidence of constitutional symptoms, such as pyrexia and vomiting, was reviewed. Severe complications, such as liver failure, liver infarction, liver abscess, and bile duct necrosis, were also reviewed.
2.7. Statistical Analysis. Statistical analysis was performed using R 2.15.1 (CRAN: the Comprehensive R Archive Network at http://cran.r-project.org/). The parameters related to the patient and nodule characteristics, details of treatment, and adverse events were assessed with the Student's $t$-test, the Mann-Whitney U-test, and the Fisher's exact test. TE grades were assessed with the Mann-Whitney $U$-test. Logistic regression analysis was performed to identify factors that had significant impact on ORR, among potential prognostic factors: sex, hepatitis B virus infection, tumor size, values of $\alpha$-fetoprotein (AFP) and des-gamma-carboxyprothrombin (DCP), Barcelona Clinic Liver Cancer (BCLC) stage, history of TACE, warming miriplatin, dose of miriplatin, and preoperative severe hepatic arterial damage [17-19]. Two-way ANOVA test was performed to reveal interaction between previous treatment history or preoperative severe arterial damage and warming miriplatin. Kappa value was calculated to assess the degree of interobserver agreement in evaluation of the hepatic arterial damage.

\section{Results}

3.1. Demographic Data and Tumor Profiles. Demographic data and parameters related to the patients and nodules are summarized in Table 1 . No significant differences in age, sex, etiology of underlying chronic liver disease, ChildPugh score, BCLC stage, or performance status (Eastern Cooperative Oncology Group classification) were observed between the nonwarmed and warmed miriplatin groups $(P=$ $0.086,0.10,0.093,0.73,0.72$, and 0.15, resp.). Preoperative AFP and DCP values were not available in 9 cases. No significant difference was observed in AFP and DCP values and nodule size (the product of long and short axes length) $(P=0.80,0.15$, and 0.72 , resp.). 
TABle 1: Profile of patients and nodules.

\begin{tabular}{|c|c|c|c|}
\hline & Nonwarmed group $(n=22)$ & Warmed group $(n=58)$ & $P$ value \\
\hline Age, years & $68(51-83)$ & $73(50-91)$ & 0.086 \\
\hline \multicolumn{4}{|l|}{ Sex, $n(\%)$} \\
\hline Male/female & $17 / 5(77.3 / 22.7 \%)$ & $34 / 24(58.6 / 41.4 \%)$ & 0.10 \\
\hline Etiology, $n(\%)$ & & & 0.093 \\
\hline $\mathrm{HCV}$ & $14(63.6 \%)$ & $44(75.9 \%)$ & \\
\hline $\mathrm{HBV}$ & $1(4.5 \%)$ & $7(12.1 \%)$ & \\
\hline Alcohol & $5(22.7 \%)$ & $3(5.2 \%)$ & \\
\hline Others & $2(9.2 \%)$ & $4(6.8 \%)$ & \\
\hline Child-Pugh, $n(\%)$ & & & 0.73 \\
\hline 5-6 (Class A) & $13(59.1 \%)$ & $33(56.9 \%)$ & \\
\hline 7-9 (Class B) & $8(36.4 \%)$ & $23(39.7 \%)$ & \\
\hline 10 (Class C) & $1(4.5 \%)$ & $2(3.4 \%)$ & \\
\hline Median score & 6.4 & 6.5 & \\
\hline BCLC stage, $n(\%)$ & & & 0.72 \\
\hline 0 (very early) & $0(0 \%)$ & $0(0 \%)$ & \\
\hline A (early) & $0(0 \%)$ & $0(0 \%)$ & \\
\hline $\mathrm{B}$ (intermediate) & $8(36.4 \%)$ & $21(36.2 \%)$ & \\
\hline $\mathrm{C}$ (advanced) & $12(54.5 \%)$ & $36(62.1 \%)$ & \\
\hline $\mathrm{D}$ (terminal) & $2(9.1 \%)$ & $1(1.7 \%)$ & \\
\hline PS, $n(\%)$ & & & 0.15 \\
\hline 0 & $6(27.3 \%)$ & $23(39.7 \%)$ & \\
\hline 1 & $13(59.1 \%)$ & $33(56.9 \%)$ & \\
\hline 2 & $2(9.1 \%)$ & $2(3.4 \%)$ & \\
\hline 3 & $1(4.5 \%)$ & $0(0 \%)$ & \\
\hline $\operatorname{AFP}(\mathrm{ng} / \mathrm{mL})$ & $282.8 \pm 723.1$ & $415.5 \pm 1454.3$ & 0.80 \\
\hline $\mathrm{DCP}(\mathrm{mAU} / \mathrm{mL})$ & $1562.6 \pm 5241.2$ & $581.3 \pm 2913.8$ & 0.15 \\
\hline Nodule size $\left(\mathrm{mm}^{2}\right)$ & $388.6 \pm 398.0$ & $383.1 \pm 468.0$ & 0.72 \\
\hline History of TACE & $18(81.8 \%)$ & $32(55.2 \%)$ & $0.038^{*}$ \\
\hline \multicolumn{4}{|l|}{ Agent } \\
\hline EPI alone & $8 / 18(44.4 \%)$ & $4 / 32(12.5 \%)$ & \\
\hline CDDP alone & $6 / 18(33.3 \%)$ & $12 / 32(37.5 \%)$ & \\
\hline MPT alone & $0 / 18(0 \%)$ & $5 / 32(15.6 \%)$ & \\
\hline Multiple agents & $4 / 18(22.3 \%)$ & $11 / 32(34.4 \%)$ & \\
\hline Number of sessions & & & 0.087 \\
\hline 1 & $11 / 18(61.1 \%)$ & $17 / 32(53.1 \%)$ & \\
\hline 2 & $4 / 18(22.2 \%)$ & $9 / 32(28.1 \%)$ & \\
\hline 3 & $1 / 18(5.6 \%)$ & $4 / 32(12.5 \%)$ & \\
\hline $4-6$ & $2 / 18(11.1 \%)$ & $2 / 32(6.3 \%)$ & \\
\hline Preoperative severe arterial damage & $7(31.8 \%)$ & $6(10.3 \%)$ & $0.037^{*}$ \\
\hline Follow-up period (months) & $8.5 \pm 7.6(2-36)$ & $7.7 \pm 6.0(2-29)$ & 0.68 \\
\hline$\geq 3$ months & $19(86.4 \%)$ & $49(84.5 \%)$ & 1.00 \\
\hline$\geq 6$ months & $11(50 \%)$ & $31(53.4 \%)$ & 0.81 \\
\hline Interval between image and TACE (months) ${ }^{\dagger}$ & & & 0.084 \\
\hline$<1$ month & $26 / 29(89.7 \%)$ & $62 / 87(71.3 \%)$ & \\
\hline $1-2$ months & $1 / 29(3.4 \%)$ & $18 / 87(20.7 \%)$ & \\
\hline 2-3 months & $2 / 29(6.9 \%)$ & $7 / 87(8.0 \%)$ & \\
\hline
\end{tabular}

Age is presented as median (range).

AFP, DCP, nodule size, and follow-up period are presented as mean \pm standard deviation.

Range of follow-up period is shown in the parentheses.

${ }^{*} P<0.05$

${ }^{\dagger}$ Interval between preoperative image and treatment was evaluated on a per treatment session basis.

HCV: hepatitis C virus; HBV: hepatitis B virus; AFP: alpha-fetoprotein; DCP: des-gamma-carboxyprothrombin; BCLC: Barcelona Clinic Liver Cancer; PS: performance status (Eastern Cooperative Oncology Group classification); TACE: transcatheter arterial chemoembolization; EPI: epirubicin; CDDP: cisplatin; MPT: miriplatin. 


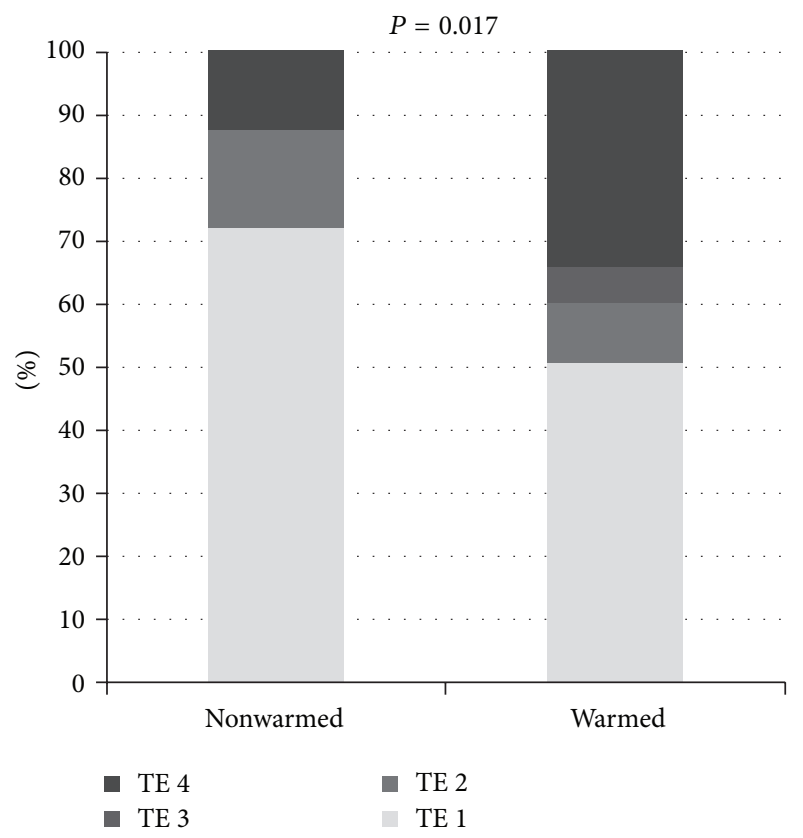

Figure 2: Treatment effect. The bar graph shows the distribution of treatment effect (TE) grades.

Eighteen cases (81.8\%) and 32 cases (55.2\%) had a previous history of TACE in the nonwarmed and warmed miriplatin groups, respectively (Table 1). Conventional lipiodol-TACE was performed using epirubicin, cisplatin, or miriplatin in previous sessions. Gelatin sponge was used as embolization material. The ratio of nodules with previous TACE history was significantly higher in the nonwarmed miriplatin group $(P=0.038)$. No significant difference was observed in the number of previous treatment sessions between the 2 groups $(P=0.087)$.

No significant difference was observed in follow-up period $(P=0.68)$. More than $80 \%$ of the nodules were followed up for more than 3 months and about half of the nodules were followed up for more than 6 months: longer follow-up period than previous studies $[20,21]$. Frequency of preoperative severe arterial damage was significantly higher in the nonwarmed group $(P=0.037)$. There was no significant difference in the interval between preoperative image evaluation and treatment between the 2 groups $(P=$ 0.084).

3.2. Treatment Effect. Warming miriplatin had an impact on TE grades. TE grades were significantly higher in the warmed miriplatin group than in the nonwarmed miriplatin group $(P=0.017$; Figure 2$)$. In the nonwarmed miriplatin group, 4 lesions were classified as TE $4(12.5 \%), 0$ as TE $3(0 \%), 5$ as TE $2(15.6 \%)$, and 23 as TE 1 (71.9\%); thus, ORR and DCR were $12.5 \%$ and $28.1 \%$, respectively. In contrast, in the warmed miriplatin group, 29 lesions were classified as TE 4 (34.1\%), 5 as TE $3(5.9 \%), 8$ as TE $2(9.4 \%)$, and 43 as TE 1 (50.6\%); thus, ORR and DCR were $40.0 \%$ and $49.4 \%$, respectively. ORR was significantly higher in the warmed group $(P=0.0042)$, while there was no significant difference in $\operatorname{DCR}(P=0.059)$.
TABLE 2: Results of logistic regression analysis.

\begin{tabular}{lcc}
\hline Factors & Odds ratio (95\% CI) & $P$ value \\
\hline Sex (female) & $0.39(0.12-1.17)$ & 0.10 \\
HBV infection & $0.30(0.046-1.49)$ & 0.16 \\
Tumor size $\left(\mathrm{mm}^{2}\right)$ & $1.00(1.00-1.00)$ & 0.13 \\
AFP $(\mathrm{ng} / \mathrm{mL})$ & $1.00(1.00-1.00)$ & 0.16 \\
DCP $(\mathrm{mAU} / \mathrm{mL})$ & $1.00(1.00-1.00)$ & 0.14 \\
BCLC stage C & $0.77(0.24-2.43)$ & 0.66 \\
BCLC stage D & $36.55(0.70-3635.77)$ & 0.078 \\
History of TACE & $0.78(0.27-2.24)$ & 0.64 \\
Warming miriplatin & $12.35(2.90-90.0)$ & $0.0028^{* *}$ \\
Miriplatin dose (mg) & $0.99(0.96-1.01)$ & 0.43 \\
Severe hepatic arterial damage & $0.59(0.10-2.73)$ & 0.52 \\
\hline ** < 0.01. & \\
CI: confidence interval; HBV: hepatitis B virus; AFP: alpha-fetoprotein; DCP: \\
des-gamma-carboxyprothrombin; BCLC: Barcelona Clinic Liver Cancer; \\
TACE: transcatheter arterial chemoembolization.
\end{tabular}

TACE using both nonwarmed miriplatin and warmed miriplatin was performed on 3 nodules. TE 1 was obtained in all cases after TACE using nonwarmed miriplatin. Treatment effect improved to TE 4 in 1 case (Figure 3); however, no improvement was observed in the other 2 nodules after TACE using warmed miriplatin. No significant difference was observed in the amount of administered miriplatin (33.5 \pm $16.7 \mathrm{mg}$ in the nonwarmed miriplatin group versus $42.9 \pm$ $29.8 \mathrm{mg}$ in the warmed miriplatin group; $P=0.18$ ).

Logistic regression analysis revealed that warming miriplatin had a significant impact on ORR (odds ratio, 12.35; 95\% confidence interval, 2.90-90.0; $P=0.0028$; Table 2). Other factors did not have a significant impact on ORR.

Since significant difference was observed in previous treatment history and preoperative severe arterial damage, two-way ANOVA test was performed to reveal interaction between these factors and warming miriplatin. No significant interaction was observed between previous treatment history and warming miriplatin $(P=0.24)$ and between preoperative severe hepatic arterial damage and warming miriplatin $(P=$ $0.38)$.

3.3. Angiographic Evaluation after TACE. Forty-three cases were included in the epirubicin/cisplatin treatment group. Thirty cases were included in the nonwarmed miriplatin group, considering 8 cases in the warmed miriplatin group with past treatment history using nonwarmed miriplatin. Postoperative angiography was not available in 12 cases in the warmed miriplatin group; therefore, 46 cases were included.

Discrepancy in arterial damage grade evaluation was observed in 7 cases, while that in arterial damage level evaluation was observed in 2 cases with the epirubicin/cisplatin group ( $\kappa$ value: 0.71 and 0.77 , resp.). Discrepancy in arterial damage grade evaluation was observed in 2 cases with both nonwarmed and warmed miriplatin groups ( $\kappa$ value: 0.85 and 0.70 , resp.). Agreement on arterial damage level was obtained in all cases with the nonwarmed and warmed miriplatin 


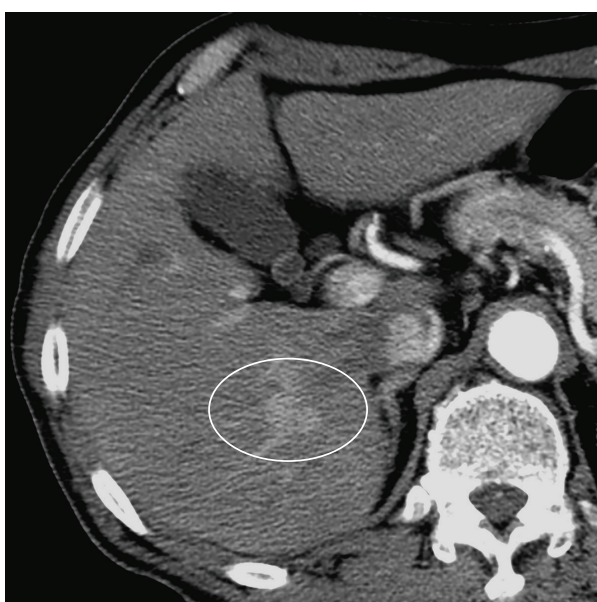

(a)

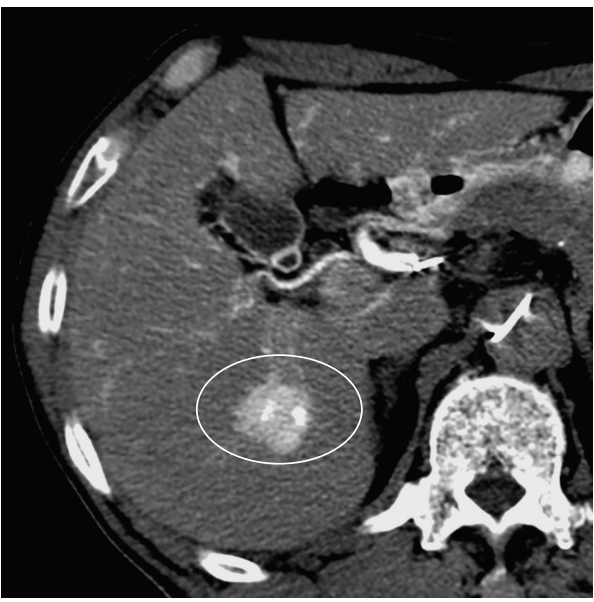

(c)

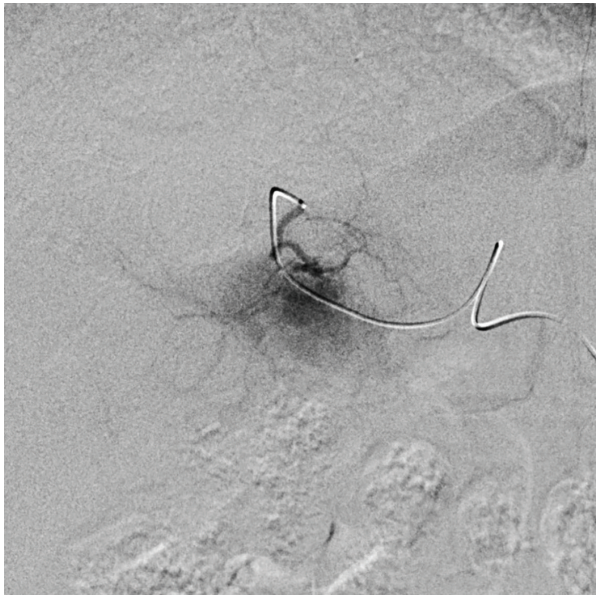

(e)

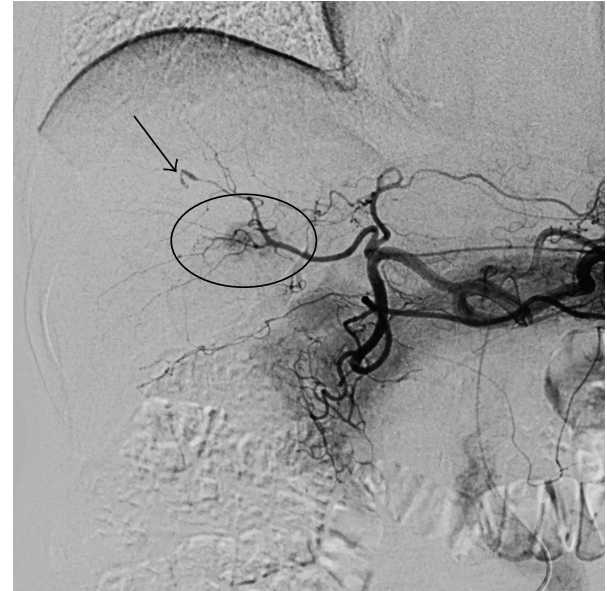

(b)

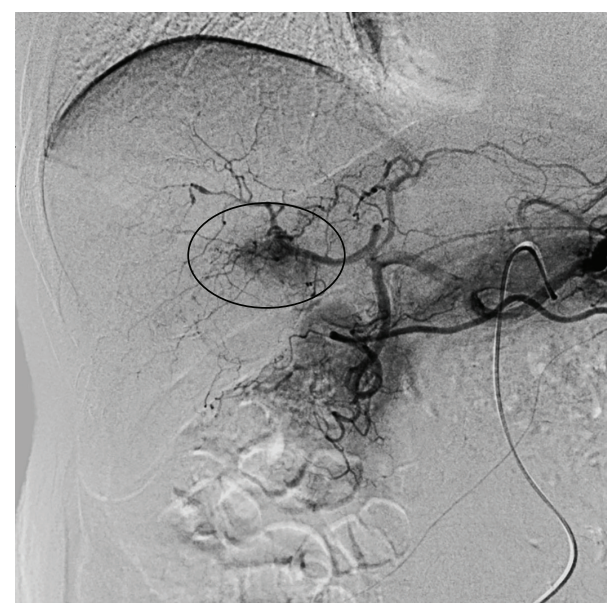

(d)

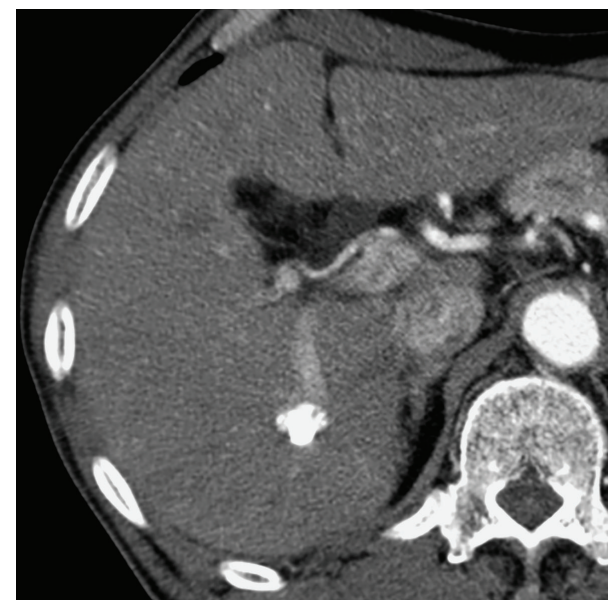

(f)

FIGURE 3: A case of hepatocellular carcinoma treated with warmed miriplatin. (a) Arterial phase of contrast-enhanced computed tomography (CT) before treatment. The white circle shows an enhanced lesion compatible with hepatocellular carcinoma. (b) Common hepatic arteriography showing a tumor stain (black circle). Occlusion and aneurysm formation are noted in the hepatic arterial branch, presumably caused by previous transcatheter arterial chemoembolization (TACE) using cisplatin (black arrow). (c) Early phase of CT during arteriography with the catheter tip placed in the common hepatic artery, 6 months after the first session of TACE using nonwarmed miriplatin. Obvious enhancement was observed, which indicated recurrence (white circle). (d) Celiac arteriography showing tumor stain (black circle). (e) Selective angiography with a microcatheter placed in a feeding artery. TACE was performed using warmed miriplatin in this session. (f) Arterial phase of contrast-enhanced CT, 4 months after the second session of TACE. Lipiodol accumulated densely in the target lesion, and the tumor size was decreased with no evidence of recurrence. 
TABLE 3: Evaluation of the effect of anticancer agents on the hepatic artery.

\begin{tabular}{|c|c|c|c|}
\hline & Epirubicin/cisplatin $(n=43)$ & Nonwarmed miriplatin $(n=30)$ & Warmed miriplatin $(n=46)$ \\
\hline \multicolumn{4}{|l|}{ Damage grade } \\
\hline 0 (no damage) & $25(58.1 \%)$ & $22(73.3 \%)$ & $44(95.7 \%)$ \\
\hline 1 (irregularity) & $5(11.6 \%)$ & $6(20.0 \%)$ & $2(4.3 \%)$ \\
\hline 2 (narrowing) & $1(2.4 \%)$ & $0(0 \%)$ & $0(0 \%)$ \\
\hline 3 (stenosis) & $3(7.0 \%)$ & $0(0 \%)$ & $0(0 \%)$ \\
\hline 4 (occlusion) & $9(20.9 \%)$ & $2(6.7 \%)$ & $0(0 \%)$ \\
\hline \multicolumn{4}{|l|}{ Damage level } \\
\hline 1 (PHA) & $1 / 18(5.6 \%)$ & $0 / 8(0 \%)$ & $0 / 2(0 \%)$ \\
\hline 2 (lobar branch) & $3 / 18(16.7 \%)$ & $0 / 8(0 \%)$ & $0 / 2(0 \%)$ \\
\hline 3 (segmental branch) & $6 / 18(33.3 \%)$ & $3 / 8(37.5 \%)$ & $1 / 2(50 \%)$ \\
\hline 4 (subsegmental branch) & $8 / 18(44.4 \%)$ & $5 / 8(62.5 \%)$ & $1 / 2(50 \%)$ \\
\hline \multicolumn{4}{|l|}{ A-P shunt formation } \\
\hline Yes & $2(4.7 \%)$ & $3(10.0 \%)$ & $0(0 \%)$ \\
\hline No & $41(95.3 \%)$ & $27(90.0 \%)$ & $46(100 \%)$ \\
\hline \multicolumn{4}{|l|}{ Number of sessions } \\
\hline 1 & $28(65.1 \%)$ & $24(80.0 \%)$ & $27(58.7 \%)$ \\
\hline 2 & $10(23.3 \%)$ & $5(16.7 \%)$ & $14(30.4 \%)$ \\
\hline 3 & $1(2.3 \%)$ & $1(3.3 \%)$ & $4(8.7 \%)$ \\
\hline $4-6$ & $4(9.3 \%)$ & $0(0 \%)$ & $1(2.2 \%)$ \\
\hline
\end{tabular}

Data in this table were obtained from consensus of two radiologists. PHA: proper hepatic artery; A-P shunt: arterioportal shunt.

groups. Agreement on A-P shunt formation was obtained in all cases with all groups. Discrepancy in the findings was discussed by two observers and consensus was formed in all cases.

According to the consensus, severe arterial damage was observed in 12 of 43 cases (27.9\%) with the epirubicin/cisplatin group, in 2 of 30 cases $(6.7 \%)$ with the nonwarmed miriplatin group, and in 0 of 46 cases $(0 \%)$ with the warmed miriplatin group (Table 3 ). There was no significant difference in the number of treatment sessions $(P=0.164)$.

3.4. Adverse Events. Severe complications, such as liver abscess, bile duct necrosis, and liver infarction, and complications above CTCAE grade 4 were not observed in either group. There was no 30-day mortality.

Grades of AST and ALT elevation after treatment were significantly higher in the warmed miriplatin group $(P=$ 0.0083 and 0.0068 , resp.; Table 4 ), although no significant difference was observed in preoperative values $(P=0.14$ and 0.32 , resp.). Return to the preoperative level within 1 month was observed in all cases, with the exception of 1 and 2 cases in the nonwarmed and warmed miriplatin groups, respectively, in which sustained mild elevation of AST and ALT (80-150 IU/L) was observed. These cases were managed conservatively.

No significant difference was observed in grades of anemia $(P=0.060$; Table 4$)$; however, transfusion was necessary in 2 cases. No obvious relationship between anemia and TACE was identified, and recovery to the preoperative level was observed in all cases within 1 month. Data on eosinophilia was missing in 2 sessions with the nonwarmed miriplatin group and in 25 sessions with the warmed miriplatin group. No significant difference was observed in occurrence of eosinophilia $(P=0.26)$. No significant difference was observed in other parameters.

\section{Discussion}

In this study, the improved efficacy of warmed miriplatin compared to nonwarmed miriplatin when used in TACE for HCC was demonstrated; both TE and ORR were improved in the warmed miriplatin group. DCR was also better in the warmed miriplatin group, although this difference was not significant. No significant difference was observed in patient profiles or parameters related to each nodule, except history of TACE and preoperative severe hepatic arterial damage, which was more frequent in the nonwarmed miriplatin group. However, logistic regression analysis revealed that these parameters had no significant impact on objective response. Two-way ANOVA tests also revealed that there was no significant interaction between these parameters and warming miriplatin. On the contrary, Seko et al. reported that history of TACE had significant impact on tumor response [20]. This discrepancy in conclusion can be attributed to the difference in evaluation method (modified RECIST versus TE), since tumor response can be evaluated differently with different criteria. Furthermore, they did not consider hepatic arterial damage, which can have direct impact on tumor response. Therefore this study gives more comprehensive 
TABLE 4: Adverse events.

\begin{tabular}{|c|c|c|c|}
\hline & Nonwarmed group (29 sessions) & Warmed group (87 sessions) & $P$ value \\
\hline AST (IU/L), $n(\%)$ & $46.8 \pm 22.4$ & $59.1 \pm 50.0$ & 0.14 \\
\hline Grade 1 & $10(34.5 \%)$ & $24(27.6 \%)$ & \\
\hline Grade 2 & $5(17.2 \%)$ & $20(23.0 \%)$ & \\
\hline Grade 3 & $3(10.3 \%)$ & $26(29.9 \%)$ & \\
\hline Grades $4-5$ & $0(0)$ & $0(0)$ & $0.0083^{* *}$ \\
\hline $\operatorname{ALT}(\mathrm{IU} / \mathrm{L}), n(\%)$ & $35.9 \pm 15.6$ & $50.6 \pm 56.2$ & 0.32 \\
\hline Grade 1 & $8(27.6 \%)$ & $27(31.0 \%)$ & \\
\hline Grade 2 & $1(3.4 \%)$ & $14(16.1 \%)$ & \\
\hline Grade 3 & $4(13.8 \%)$ & $22(25.3 \%)$ & \\
\hline Grades $4-5$ & $0(0)$ & $0(0)$ & $0.0068^{* *}$ \\
\hline T-Bil (mg/dL), $n(\%)$ & $1.03 \pm 0.75$ & $0.81 \pm 0.39$ & 0.25 \\
\hline Grade 1 & $5(17.2 \%)$ & $29(33.3 \%)$ & \\
\hline Grade 2 & $3(10.3 \%)$ & $8(9.2 \%)$ & \\
\hline Grade 3 & $0(0)$ & $0(0)$ & \\
\hline Grades $4-5$ & $0(0)$ & $0(0)$ & 0.22 \\
\hline $\mathrm{WBC}(/ \mu \mathrm{L}), n(\%)$ & $3666 \pm 1477$ & $3840 \pm 1604$ & 0.59 \\
\hline Grade 1 & $1(3.4 \%)$ & $7(8.0 \%)$ & \\
\hline Grade 2 & $1(3.4 \%)$ & $7(8.0 \%)$ & \\
\hline Grade 3 & $3(10.3 \%)$ & $9(10.3 \%)$ & \\
\hline Grades 4-5 & $0(0)$ & $0(0)$ & 0.38 \\
\hline $\mathrm{Hb}(\mathrm{g} / \mathrm{dL}), n(\%)$ & $12.0 \pm 2.0$ & $11.6 \pm 1.9$ & 0.32 \\
\hline Grade 1 & $3(10.3 \%)$ & $19(21.8 \%)$ & \\
\hline Grade 2 & $1(3.4 \%)$ & $10(11.5 \%)$ & \\
\hline Grade 3 & $1(3.4 \%)$ & $3(3.4 \%)$ & \\
\hline Grades $4-5$ & $0(0)$ & $0(0)$ & 0.060 \\
\hline Plt $\left(\times 10^{4} / \mu \mathrm{L}\right), n(\%)$ & $9.2 \pm 4.5$ & $10.8 \pm 5.0$ & 0.20 \\
\hline Grade 1 & $5(17.2 \%)$ & $26(29.9 \%)$ & \\
\hline Grade 2 & $7(24.1 \%)$ & $19(21.8 \%)$ & \\
\hline Grade 3 & $2(6.9 \%)$ & $14(16.1 \%)$ & \\
\hline Grades 4-5 & $0(0)$ & $0(0)$ & 0.11 \\
\hline Eosinophilia $^{\dagger}$ & $13 / 27(48.1 \%)$ & $22 / 62(35.5 \%)$ & 0.26 \\
\hline \multicolumn{4}{|l|}{ Pyrexia, $n(\%)$} \\
\hline Grade 1 & $11(37.9 \%)$ & $35(40.2 \%)$ & \\
\hline Grade 2 & $0(0)$ & $7(8.0 \%)$ & \\
\hline Grades 3-5 & $0(0)$ & $0(0)$ & 0.22 \\
\hline \multicolumn{4}{|l|}{ Vomiting, $n(\%)$} \\
\hline Grade 1 & $1(3.4 \%)$ & $3(3.4 \%)$ & \\
\hline Grade 2 & $1(3.4 \%)$ & $0(0)$ & \\
\hline Grades 3-5 & $0(0)$ & $0(0)$ & 0.42 \\
\hline Liver infarction & 0 & 0 & - \\
\hline Liver abscess & 0 & 0 & - \\
\hline Bile duct necrosis & 0 & 0 & - \\
\hline
\end{tabular}

Preoperative values of AST, ALT, T-Bil, WBC, Hb, and Plt are presented as mean \pm standard deviation in the first row.

$P$ values in the first row are for comparison of preoperative values, while values in the bottom row are for comparison of CTCAE grades.

${ }^{* *} P<0.01$

${ }^{\dagger}$ Data on eosinophilia were missing in 2 sessions with the nonwarmed group and in 25 sessions with the warmed group.

AST: aspartate transaminase; ALT: alanine transaminase; T-Bil: total bilirubin; WBC: white blood cell; Hb: hemoglobin; Plt: platelets.

analysis; however, prospective trial with matched background is desirable to obtain appropriate conclusion.

TACE-induced hepatic arterial damage is a factor that limits the efficacy of this treatment. Good interobserver agreement was obtained in evaluation of the hepatic arterial damage grade and level. Severe arterial damage, which can interfere with injection of anticancer agents and lipiodol, was less frequent with nonwarmed and warmed miriplatin, compared to epirubicin/cisplatin (6.7\% and $0 \%$ versus $27.9 \%)$. More proximal arterial damage was observed with epirubicin/cisplatin. These facts suggest that miriplatin is more suitable for TACE due to its less severe arterial insult.

However, previous studies have shown inferior local tumor control of TACE using miriplatin compared to other agents, including cisplatin and epirubicin [11-13]. Iwazawa et al. reported that the high viscosity of miriplatin suspension 
may result in early occlusion of tumor feeders before sufficient accumulation of miriplatin in the tumor is obtained, and this may be a major factor related to inferior local tumor control [11]. The viscosity of miriplatin has been shown to decrease with increasing temperature $[14,15]$; a few studies have revealed improved local tumor control when investigating the efficacy of TACE using warmed miriplatin $[20,21]$. However, these studies showed only the short-term TE (less than 3 months); in contrast, our study investigated the longer-term TE ( 8.5 and 7.7 months in average with the nonwarmed and warmed miriplatin groups, resp.). Another advantage of our study is its practical study design; nodules were followed up until treatments other than miriplatinTACE were performed. Thus, the efficacy of miriplatin-TACE was genuinely evaluated.

The reason why local tumor control can be improved when using warmed miriplatin remains unclear. According to the hypothesis that agents with high viscosity cause proximal occlusion of feeding arteries, warmed miriplatin, which is therefore less viscous, can be injected to more distal parts of feeding arteries. Distal vessels usually have more vascular beds than proximal vessels, and thus the amount of injected miriplatin suspension may be assumed to increase; however, there was no significant difference in the miriplatin dose between the nonwarmed and warmed miriplatin groups in our study. Additional studies should be performed to reveal the mechanism underlying the improved efficacy of TACE using warmed miriplatin.

Despite the promising results, local tumor control in this study was not comparable to that found in previous studies on TACE using miriplatin [7,9-12]. This may be attributed to the profile of cases included in this study. The levels of tumor markers investigated in our study (AFP and DCP) were quite high, and these markers are known to be negative prognostic factors given their association with aggressive pathological features [22-24]. Previous clinical studies have also shown that cases with high AFP levels have poor prognoses $[25,26]$.

With regard to adverse events, CTCAE grades of AST and ALT elevation were significantly higher in the warmed group. There is no consensus on whether elevation of transaminase levels after treatment is caused by damage to normal liver parenchyma or tumor necrosis, so it is difficult to interpret these data. Elevation of transaminase levels was only transient and was managed conservatively. No significant difference was observed in other parameters. Moreover, no serious complications, such as liver infarction, liver abscess, and bile duct necrosis, were observed in either group. These findings indicate that adverse events of TACE using warmed miriplatin are only transient.

Our findings provide evidence that warmed miriplatin can improve the efficacy of TACE; however, this study has some limitations. First, it is a retrospective study, and a randomized controlled study should be considered to further support these findings. There was asymmetry in the numbers of nodules treated in the nonwarmed and warmed groups, which was inevitable given the nature of a retrospective study. This study presented preliminary results on the efficacy of TACE using warmed miriplatin, and a prospective clinical trial is going to be conducted. Second, in order to establish the advantage of using miriplatin, the relationship between survival benefit and repeatability of TACE should be demonstrated. Moreover, the efficacy of TACE using warmed miriplatin should be compared with TACE using other agents or microspheres.

\section{Conclusions}

This study demonstrated the safety and improved efficacy of TACE using warmed miriplatin compared to nonwarmed miriplatin for the treatment of HCC.

\section{Conflict of Interests}

The authors declare that there is no conflict of interests regarding the publication of this paper.

\section{References}

[1] J. M. Llovet, C. Brú, and J. Bruix, "Prognosis of hepatocellular carcinoma: the BCLC staging classification," Seminars in Liver Disease, vol. 19, no. 3, pp. 329-338, 1999.

[2] C. M. Lo, H. Ngan, W. K. Tso et al., "Randomized controlled trial of transarterial Lipiodol chemoembolization for unresectable hepatocellular carcinoma," Hepatology, vol. 35, no. 5, pp. 1164$1171,2002$.

[3] J. M. Llovet, M. I. Real, X. Montaña et al., "Arterial embolisation or chemoembolisation versus symptomatic treatment in patients with unresectable hepatocellular carcinoma: a randomised controlled trial," The Lancet, vol. 359, no. 9319, pp. 1734-1739, 2002.

[4] A. L. Lewis, M. V. Gonzalez, A. W. Lloyd et al., "DC bead: in vitro characterization of a drug-delivery device for transarterial chemoembolization," Journal of Vascular and Interventional Radiology, vol. 17, part 1, no. 2, pp. 335-342, 2006.

[5] S. Sahara, N. Kawai, M. Sato et al., "Prospective evaluation of transcatheter arterial chemoembolization (TACE) with multiple anti-cancer drugs (epirubicin, cisplatin, mitomycin c, 5-fluorouracil) compared with TACE with epirubicin for treatment of hepatocellular carcinoma," Cardiovascular and Interventional Radiology, vol. 35, no. 6, pp. 1363-1371, 2012.

[6] M. Hanada, A. Baba, Y. Tsutsumishita et al., "Intra-hepatic arterial administration with miriplatin suspended in an oily lymphographic agent inhibits the growth of tumors implanted in rat livers by inducing platinum-DNA adducts to form and massive apoptosis," Cancer Chemotherapy and Pharmacology, vol. 64 , no. 3, pp. 473-483, 2009.

[7] T. Okusaka, S. Okada, T. Nakanishi, S. Fujiyama, and Y. Kubo, "Phase II trial of intra-arterial chemotherapy using a novel lipophilic platinum derivative (SM-11355) in patients with hepatocellular carcinoma," Investigational New Drugs, vol. 22, no. 2, pp. 169-176, 2004.

[8] J. Iwazawa, N. Hashimoto, S. Ohue, O. Muramoto, and T. Mitani, "Chemoembolization-induced arterial damage: evaluation of three different chemotherapeutic protocols using epirubicin and miriplatin," Hepatology Research, vol. 44, pp. 201-208, 2014.

[9] S. Oguro, S. Hashimoto, T. Tanaka et al., "Short-term therapeutic effects of transcatheter arterial chemoembolization using miriplatin-lipiodol suspension for hepatocellular carcinoma," Japanese Journal of Radiology, vol. 30, no. 9, pp. 735-742, 2012. 
[10] K. Okabe, T. Beppu, K. Haraoka et al., "Safety and shortterm therapeutic effects of miriplatin-lipiodol suspension in transarterial chemoembolization (TACE) for hepatocellular carcinoma," Anticancer Research, vol. 31, no. 9, pp. 2983-2988, 2011.

[11] J. Iwazawa, S. Ohue, N. Hashimoto, and T. Mitani, "Local tumor progression following lipiodol-based targeted chemoembolization of hepatocellular carcinoma: a retrospective comparison of miriplatin and epirubicin," Cancer Management and Research, vol. 4, no. 1, pp. 113-119, 2012.

[12] S. Miyayama, M. Yamashiro, Y. Shibata et al., "Comparison of local control effects of superselective transcatheter arterial chemoembolization using epirubicin plus mitomycin $\mathrm{C}$ and miriplatin for hepatocellular carcinoma," Japanese Journal of Radiology, vol. 30, no. 3, pp. 263-270, 2012.

[13] T. Handa, Y. Imai, K. Sugawara et al., "Transcatheter arterial chemoembolization for hepatocellular carcinoma: comparison of the therapeutic efficacies between miriplatin and epirubicin," Hepatology Research, 2013.

[14] S. Murata, T. Mine, T. Ueda et al., "Transcatheter arterial chemoembolization based on hepatic hemodynamics for hepatocellular carcinoma," The Scientific World Journal, vol. 2013, Article ID 479805, 8 pages, 2013.

[15] T. Hasegawa, H. Takaki, T. Yamanaka et al., "Experimental assessment of temperature influence on miriplatin and cisplatin iodized-oil suspension viscosity," Japanese Journal of Radiology, vol. 31, no. 6, pp. 424-427, 2013.

[16] M. Kudo, S. Kubo, K. Takayasu et al., "Response Evaluation Criteria in Cancer of the Liver (RECICL) proposed by the Liver Cancer Study Group of Japan (2009 Revised Version)," Hepatology Research, vol. 40, no. 7, pp. 686-692, 2010.

[17] J. Xiao, G. Li, S. Lin et al., "Prognostic factors of hepatocellular carcinoma patients treated by transarterial chemoembolization," International Journal of Clinical and Experimental Pathology, vol. 7, no. 3, pp. 1114-1123, 2014.

[18] K. Yamakado, S. Miyayama, S. Hirota et al., "Subgrouping of intermediate-stage (BCLC stage B) hepatocellular carcinoma based on tumor number and size and Child-Pugh grade correlated with prognosis after transarterial chemoembolization," Japanese Journal of Radiology, vol. 32, no. 5, pp. 260-265, 2014.

[19] M. T. Sellers, S. Huggins, K. Kegley et al., "Multivariate analysis of prognostic factors for survival following doxorubicin-eluting bead transarterial chemoembolization for hepatocellular carcinoma," Journal of Vascular and Interventional Radiology, vol. 24, no. 5, pp. 647-654, 2013.

[20] Y. Seko, K. Ikeda, Y. Kawamura et al., "Antitumor efficacy of transcatheter arterial chemoembolization with warmed miriplatin in hepatocellular carcinoma," Hepatology Research, vol. 43, no. 9, pp. 942-949, 2013.

[21] S.-I. Kora, H. Urakawa, T. Mitsufuji, A. Osame, H. Higashihara, and K. Yoshimitsu, "Warming effect on miriplatin-lipiodol suspension as a chemotherapeutic agent for transarterial chemoembolization for hepatocellular carcinoma: preliminary clinical experience," Cardiovascular and Interventional Radiology, vol. 36, no. 4, pp. 1023-1029, 2013.

[22] S.-Y. Peng, W. J. Chen, P.-L. Lai, Y.-M. Jeng, J.-C. Sheu, and H.-C. Hsu, "High $\alpha$-fetoprotein level correlates with high stage, early recurrence and poor prognosis of hepatocellular carcinoma: significance of hepatitis virus infection, age, p53 and $\beta$-catenin mutations," International Journal of Cancer, vol. 112, no. 1, pp. 44-50, 2004.
[23] K. Yamamoto, H. Imamura, Y. Matsuyama et al., "AFP, AFP-L3, DCP, and GP73 as markers for monitoring treatment response and recurrence and as surrogate markers of clinicopathological variables of HCC," Journal of Gastroenterology, vol. 45, no. 12, pp. 1272-1282, 2010.

[24] T. Suehiro, K. Sugimachi, T. Matsumata, H. Itasaka, A. Taketomi, and T. Maeda, "Protein induced by vitamin $\mathrm{K}$ absence or antagonist II as a prognostic marker in hepatocellular carcinoma. Comparison with alpha-fetoprotein," Cancer, vol. 73, no. 10, pp. 2464-2471, 1994.

[25] S. Savastano, D. Miotto, G. Casarrubea, S. Teso, M. ChiesuraCorona, and G. P. Feltrin, "Transcatheter arterial chemoembolization for hepatocellular carcinoma in patients with child's grade A or B cirrhosis: a multivariate analysis of prognostic factors," Journal of Clinical Gastroenterology, vol. 28, no. 4, pp. 334-340, 1999.

[26] L. Lladó, J. Virgili, J. Figueras et al., "A prognostic index of the survival of patients with unresectable hepatocellular carcinoma after transcatheter arterial chemoembolization," Cancer, vol. 88, no. 1, pp. 50-57, 2000. 


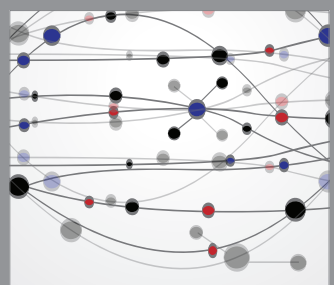

The Scientific World Journal
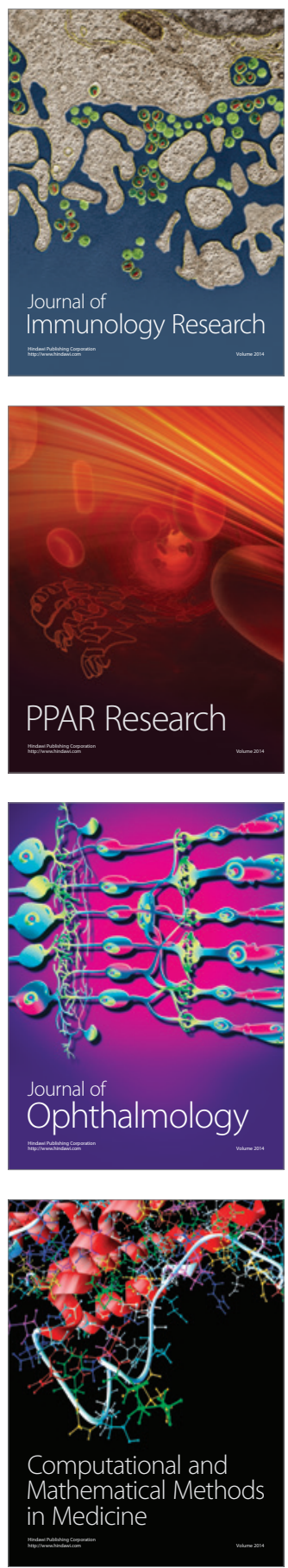

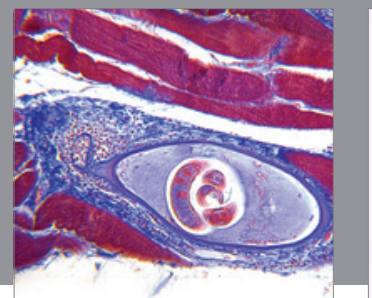

Gastroenterology

Research and Practice
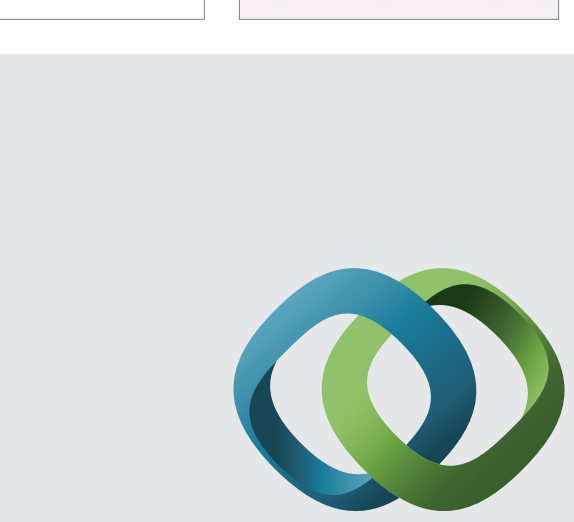

\section{Hindawi}

Submit your manuscripts at

http://www.hindawi.com
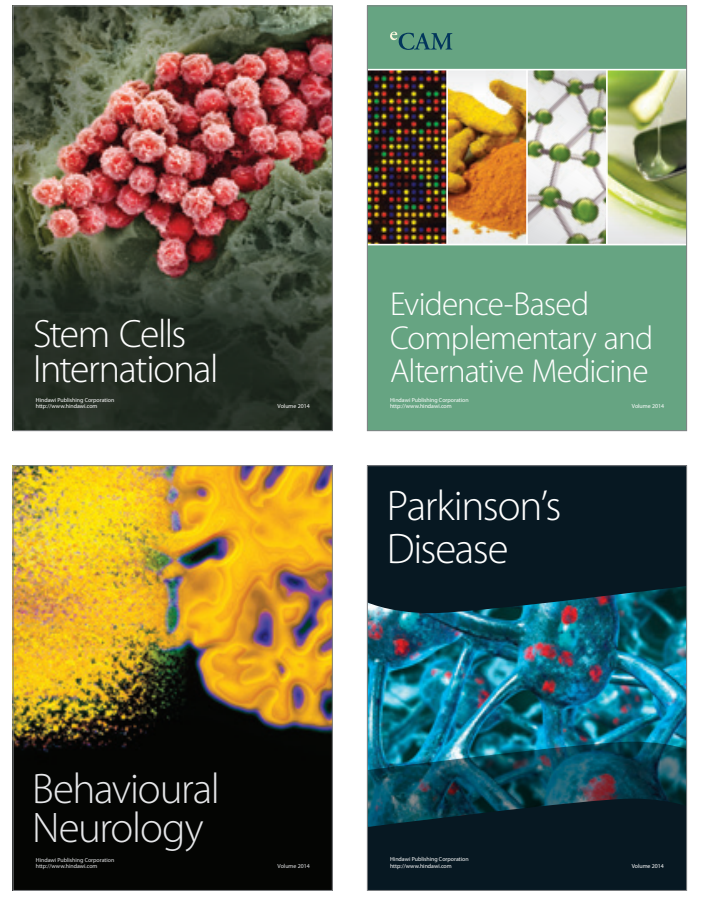
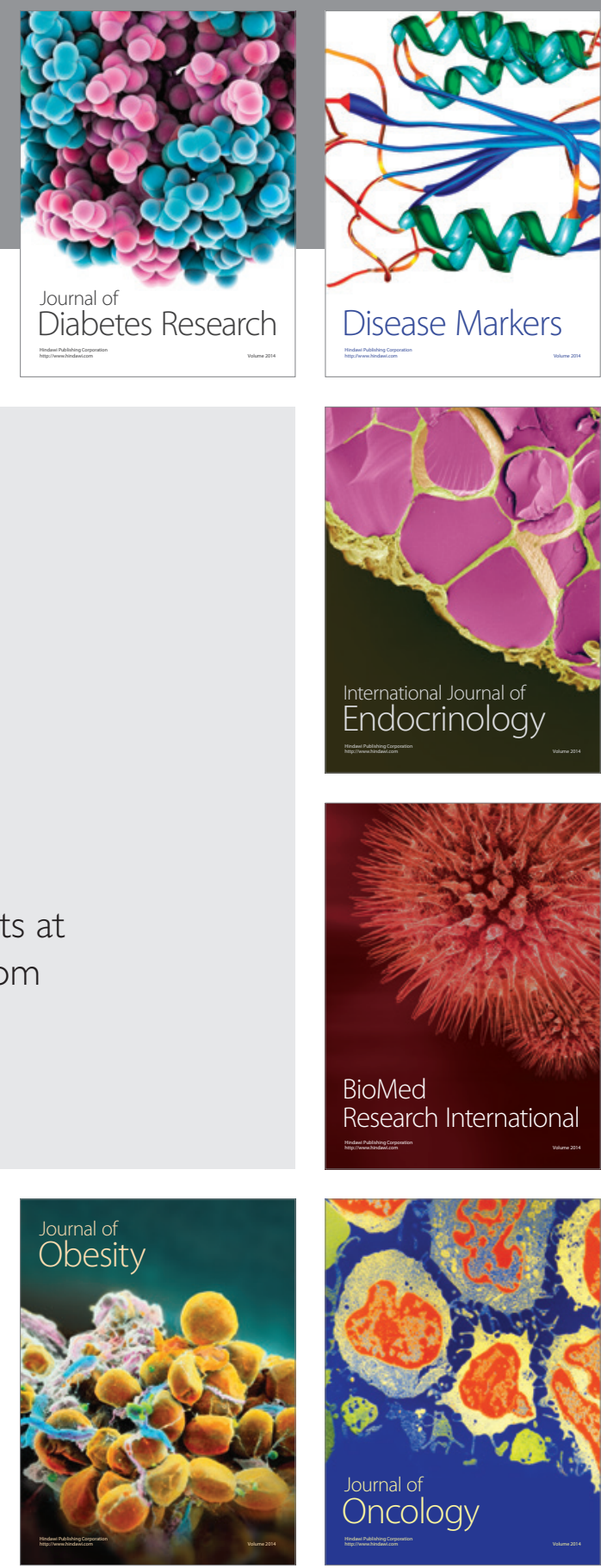

Disease Markers
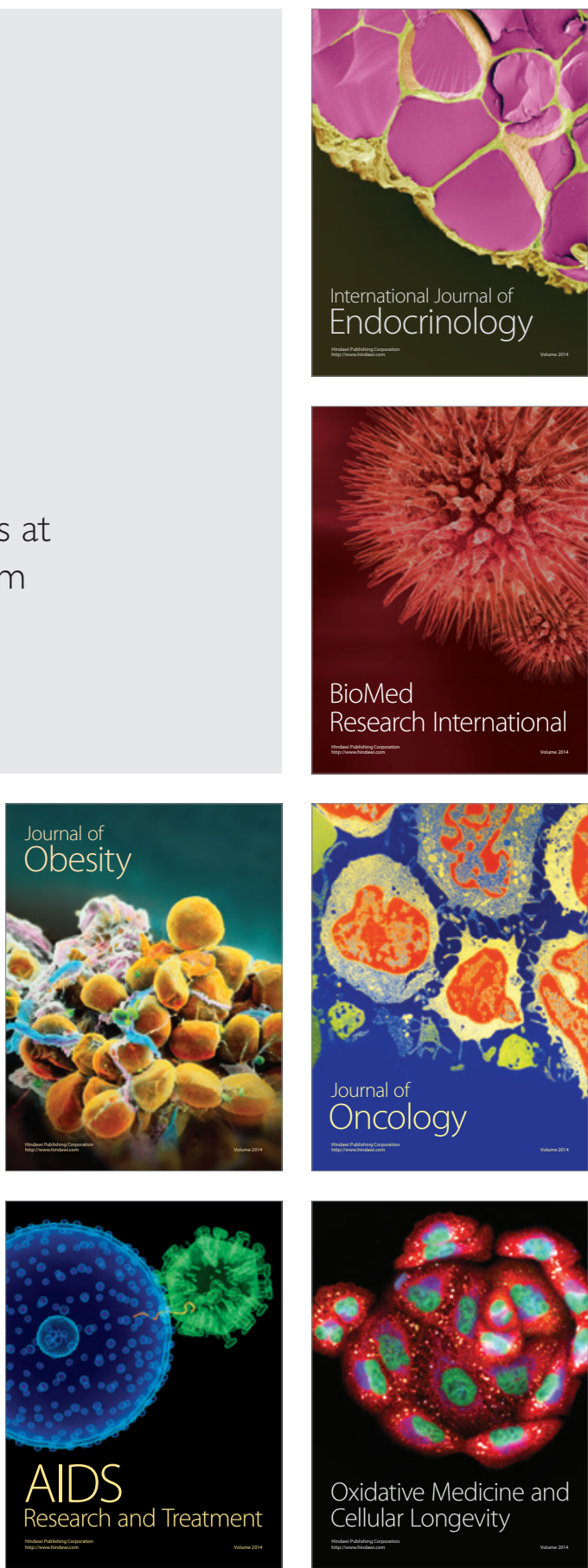